\title{
Classification of All-Rounders in the Game of ODI Cricket: Machine Learning Approach
}

\author{
By Indika Wickramasinghe*
}

\begin{abstract}
Player classification in the game of cricket is very important, as it helps the coach and the captain of the team to identify each player's role in the team and assign responsibilities accordingly. The objective of this study is to classify allrounders into one of the four categories in one day international (ODI) Cricket format and to accurately predict new all-rounders'. This study was conducted using a collection of 177 players and ten player-related performance indicators. The prediction was conducted using three machine learning classifiers, namely Naive Bayes (NB), k-nearest neighbours (kNN), and Random Forest (RF). According to the experimental outcomes, $R F$ indicates significantly better prediction accuracy of $99.4 \%$, than its counter parts.
\end{abstract}

Keywords: Team sport, machine learning, cricket, ODI, player classification

\section{Introduction}

Cricket is considered as a bat and ball team game. The game has basically three formats, namely, the test cricket, one-day-international cricket (ODI), and T20. Test cricket, the longest format is regarded by experts of the game as the ultimate test of playing skills. An ODI cricket game is played for 300 legal deliveries (balls) per side, and the shortest format, T20 is played for 120 legal deliveries (balls) per side. A typical cricket team comprises of 11 players and the team batting first is identified by the outcome of tossing a coin. In the game of cricket, there are three major disciplines: batting, bowling, and the fielding. When selecting 11 players for a team, it is necessary to balancing the team by selecting players to represent each of the above three departments.

A player who excels in bowling the cricket ball is considered as a bowler, while a player with higher potential of hitting the cricket ball is considered as a batsman. An all-rounder is a regular performer with bat and the ball. According to Bailey (1989), an all-rounder is a player who is able to grasp a position in his team for either his batting or his bowling ability. Though fielding is an integral part in the game, batting and bowling skills are given higher priorities than fielding. A genuine all-rounder is a special all-rounder who is equally capable of batting and bowling, most importantly this player can bat as a quality batsman and bowl as $\mathrm{s}$ quality bowler. Majority of all-rounders in the game of cricket dominate either batting or bowling skills, therefore they are named as batting all-rounders or as bowling all-rounders.

Identification of all-rounders is very vital for the success of a team. Classifying an all-rounder as genuine, batting, or a bowling is even beneficial for cricket selection panels, coaches, and players. A review at the literature provides evidences

*Assistant Professor, Department of Mathematics, Prairie View A\&M University, USA. 
of such studies. Using Indian Premier League (IPL) data, Saikia and Bhattacharjee (2011) classified all-rounders into four groups, namely performer, batting allrounder, bowling all-rounder, and under-performer. According to their results, the Naïve Bayes algorithm has given a classification accuracy of $66.7 \%$. In an attempt to rank all-rounders in test cricket, Tan and Ramachandran (2010) utilized both batting and bowling statistics to devise a mathematical formula. In another study, Stevenson and Brewer (2019) derived a Bayesian parametric model to predict how international cricketers' abilities change between innings in a game. Furthermore, Christie (2012) researched physical requirements of fast bowlers and stated the necessity of physiological demands to evaluate bowlers' performances. Saikia et al. (2016) developed a performance measurement using a combination of batting and bowling statistics to quantify all-rounder's performance. Wickramasinghe (2014) introduced an algorithm to predict batsman's performance using a hierarchical linear model. This multi-level model used player-level and team-level performance indicators to predict the player's performance.

Selecting a team against a given opposition team is not an easy task, as various aspects including the strengths and the weaknesses of both teams are required to consider. Bandulasiri et al. (2016) identified a typical ODI game as a mixture of batting, bowling, and decision-making. Presence of a quality allrounder in a team is an asset to a team, as it brings huge flexibility in the composition of the team. A good all-rounder makes the captain's job easy as the player can play a dual role, whenever the captain requires (Van Staden 2008). Though the impact of all-rounders towards the success of a team is enormous, there are no underline criteria to identify them.

The existence of prior research work in identifying all-rounders in the game of cricket is handful. According to the knowledge of the author, there is no existing study regarding classification of all-rounders in ODI format. Our objective of this study is to device a method to categorize all-rounders in the ODI format of cricket. We use several machine learning techniques to classify an allrounder as a genuine all-rounder, batting all-rounder, bowling all-rounder, and as an average all-rounder.

This study brings novelty for the cricket literature in many ways. According to the author's point of view, this is one of the first studies conducted to classify all-rounders in ODI version of the game using machine learning techniques. Furthermore, the selected player-related performance indicators and the used machine learning techniques are unique for this study. Findings of this study can benefit the entire cricket community and cricket industry as always prediction in sports brings an economical value to the industry (Gakis et al. 2016).

The rest of the manuscript is organized as follows. Next section will discuss about the data selection procedure and descriptive statistics about the collected data. In the methodology section, three machine learning techniques are discussed. Then, in the following section findings of this study are illustrated. Finally, the discussion and conclusion section will discuss further about the conducted study and concludes the manuscript. 


\section{Data Collection and Player - Selection Criteria}

Data for this study was collected using a publically available website, under the following criteria. Players, who have played more than 50 ODI games with an aggregate score of over 500 runs, were selected. Furthermore, it was essential for each player to have at least a half-century under their name, and collected more than 25 ODI wickets. Under the above criteria, a total of 177 players were selected and ten player related performance indicators (features) were recorded. Table 1 summarises these ten features and their descriptive statistics.

Table 1. Descriptive Statistics of Dataset

\begin{tabular}{|l|l|c|c|}
\hline Variable & \multicolumn{1}{|c|}{ Description } & Mean & SD \\
\hline Matches: & The number of games each player has played & 146.64 & 83.12 \\
\hline Runs: & $\begin{array}{l}\text { Number of accumulated runs a player has } \\
\text { scored in his career }\end{array}$ & $2,972.79$ & $2,859.03$ \\
\hline HS: & Highest score a player has scored in his career & 101.25 & 36.97 \\
\hline BatAv: & Batting average of a player & 26.04 & 9.12 \\
\hline NumCen: & $\begin{array}{l}\text { Number of times a player has scored 100 runs } \\
\text { or more in a game }\end{array}$ & 2.96 & 6.00 \\
\hline NumWkts: & $\begin{array}{l}\text { Number of accumulated wickets a player has } \\
\text { taken in this career }\end{array}$ & 115.79 & 86.05 \\
\hline BesstB: & Best bowling figures as a bowler & 4.51 & 0.98 \\
\hline BowAv: & Bowling average of the bowler & 35.04 & 6.64 \\
\hline NFiveWkts: & $\begin{array}{l}\text { Number of times a bowler has taken 5 or more } \\
\text { wickets in a game }\end{array}$ & 0.99 & 1.58 \\
\hline NCatches: & $\begin{array}{l}\text { Number of catches a player has caught in his } \\
\text { career }\end{array}$ & 47.32 & 30.83 \\
\hline
\end{tabular}

Saikia and Bhattacharjee (2011) classified all-rounders based on median value of both batting average and bowling averages. In this collected data, the distributions of both batting and bowling follow Gaussian distributions. Therefore, in this study we use the mean values of both batting and bowling averages to classify all-rounders according to the scheme summarised in Table 2. Figure 1 illustrates the joint distribution of batting and bowling averages, and the four categories of players.

Based on the Table 2 and Figure 1, we classify each all-rounder into one of the four categories: genuine all-rounder $(G)$, batting all-rounder $(B)$, bowling allrounder $(B w)$, and average all-rounder $(A)$. 
Figure 1. Distribution of Batting Averages and Bowling Averages

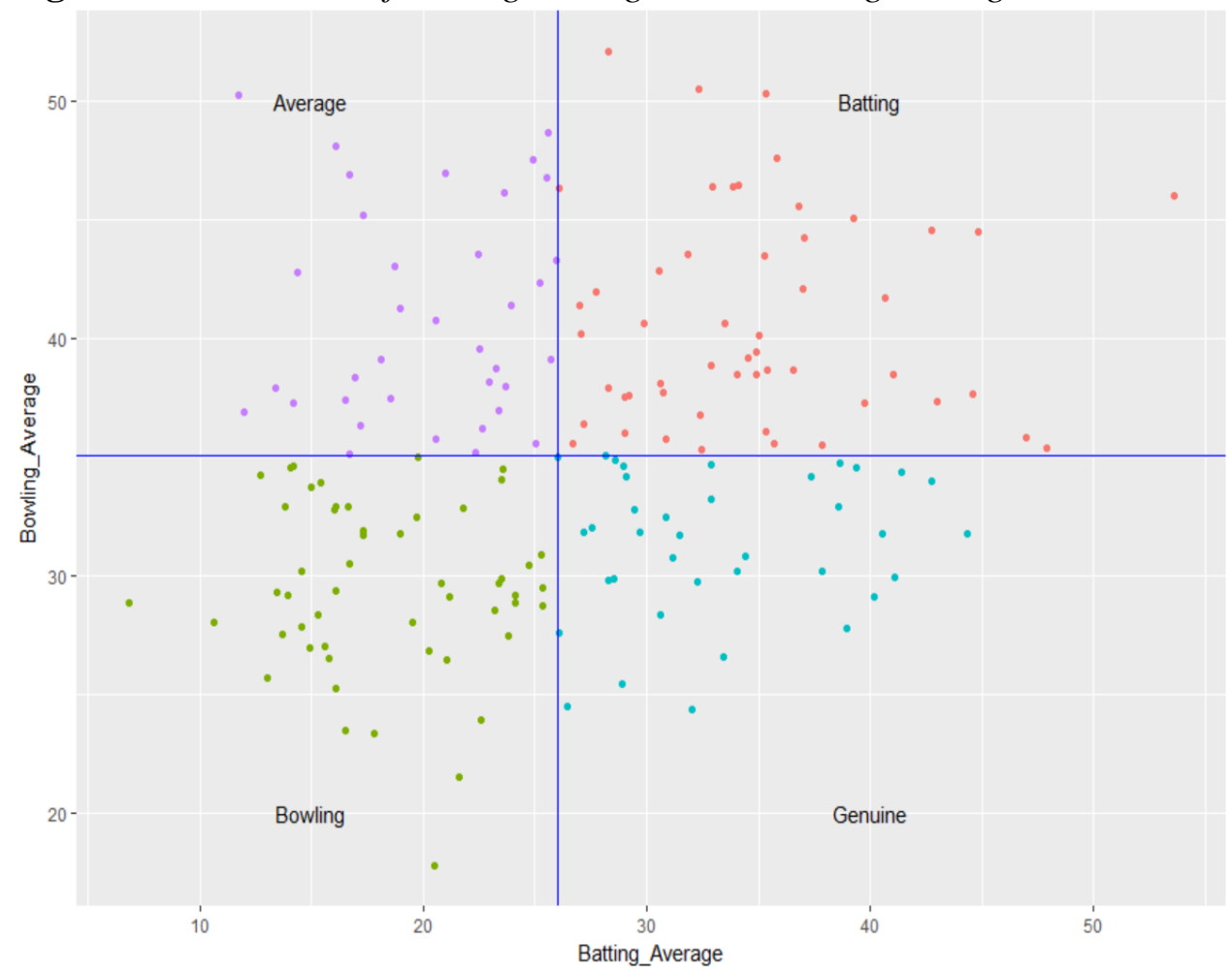

Table 2. Classification Criteria of All-Rounders

\begin{tabular}{|l|c|c|}
\hline \multirow{2}{*}{$\begin{array}{l}\text { Category of the all-rounder } \\
\text { (Type) }\end{array}$} & \multicolumn{2}{|c|}{ Criteria } \\
\hline Genuine (G) & Batting Average & Bowling Average \\
\hline Batting (B) & $>26.04$ & $<35.04$ \\
\hline Bowling (Bw) & $>26.04$ & $>35.04$ \\
\hline Average (A) & $<26.04$ & $>35.04$ \\
\hline
\end{tabular}

The class variable of the data set is named as Type, which represents each of the four classifications.

\section{Methodology}

In this study, we use three machine learning techniques, $\mathrm{NB}, \mathrm{kNN}$, and $\mathrm{RF}$ to classify all-rounders into one of the four groups. Regression analysis is one of the alternative conventional statistical procedures for an analysis like this. The number of data appoints used in regression analysis is higher, proportional to the number of involved features (Allision 1999, Bai and Pan 2009). Furthermore, some of the machine learning algorithms such as NB is considered as a better performer with smaller datasets (Hand 1992, Kuncheva 2006). Under the previously stated constraints, we opt to use these three machine learning approaches to analyse these data. 
Nä̈ve Bayes (NB)

The NB classifier is considered as one of the simplest and accurate data classifying algorithms. The base of this classifier is the well-known Bayes theorem, used in probability theory. The simplicity, the accuracy, and the robustness of NB have made NB a popular classifying technique with various applications (Arar and Ayan 2017). As the literature indicates, NB is one of the top performing classifiers used in data mining (Wu et al. 2008).

Let $X=\left(x_{1}, x_{2}, \ldots, x_{n}\right)$ be a $n$-dimensional random vector (features) from domain $D_{X}$ and $Y=\left(y_{1}, y_{2}, \ldots ., y_{m}\right)$ be a $m$-dimensional vector (classes) from domain $D_{Y}$. In this study, $n=10$ is the number of factors and $x_{1}, x_{2}, \ldots, x_{10}$, the first column of the Table 1. Similarly, here $m=4$ and $Y=\left(y_{1}, y_{2}, y_{3}, y_{4}\right) ; y_{1}=$ Genuine all - rounder, $y_{2}=$ Batting all-rounder, $y_{3}=$ Bowling all-rounder, $y_{4}=$ Average all-rounder . Our aim is to estimate the value of $Y$ by maximizing $P(Y=y \mid X=x)$. NB assumes that features are independent of each other for a given class. Therefore,

$$
\begin{aligned}
& P\left(X_{1}=x_{1}, X_{2}=x_{2}, \ldots, X_{n}=x_{n} \mid Y=y\right) \\
& =P\left(X_{1}=x_{1} \mid Y=y\right) . P\left(X_{2}=x_{2} \mid Y=y\right) \ldots P\left(X_{n}=x_{n} \mid Y=y\right) \\
& =\prod_{i=1}^{n} P\left(X_{i}=x_{i} \mid Y=y\right)
\end{aligned}
$$

According to the Bayes theorem, we have $P(y \mid X)=\frac{P(X \mid y) P(y)}{P(X)}$.

Then we can write $P(y \mid X)$ as follows.

$$
\begin{aligned}
P(y \mid X) & =\frac{P(X=x, Y=y)}{P(X=x)} \\
& =\frac{P(Y=y) P(X=x \mid Y=y)}{P(X=x)} \\
& =\frac{P(Y=y) \prod_{i=1}^{n} P\left(X_{i}=x_{i} \mid Y=y\right)}{\prod_{i=1}^{n} P\left(X_{i}=x_{i}\right)} \\
& \propto P(Y=y) \prod_{i=1}^{n} P\left(X_{i}=x_{i} \mid Y=y\right)
\end{aligned}
$$

Therefore, our aim is to find $y$, that maximize the above expression. In another words, we need to find $\mathrm{y}$, which is $\arg \max _{y} P(Y-y) P(Y=y) \prod_{i=1}^{n} P\left(X_{i}=x_{i} \mid Y=y\right)$ 


\section{k-Nearest Neighbour's Algorithm (kNN)}

The $\mathrm{kNN}$ can be considered as one of the simplest machine learning classifiers, which is based on distance matric (Figure 2). Applications of kNN can be found in text categorization (Elnahrawy 2002), ranking models (Xiubo et al. 2008), and object recognition (Bajramovic et al. 2006). If a novel data point is given, $\mathrm{kNN}$ attempts to identify the correct category of the novel point, using the characteristics of the neighbouring data points. The main trait of the data points is going to be the distance from novel data point to each of the other data points. When considering the distance metric, Euclidian is the most commonly used one though other metrics such as Manhattan Distance, Mahalanobis Distance and Chebychev Distances are also used in practice. Table 3 shows some other popular distance matrices used in data classification.

Figure 2. kNN Classifier

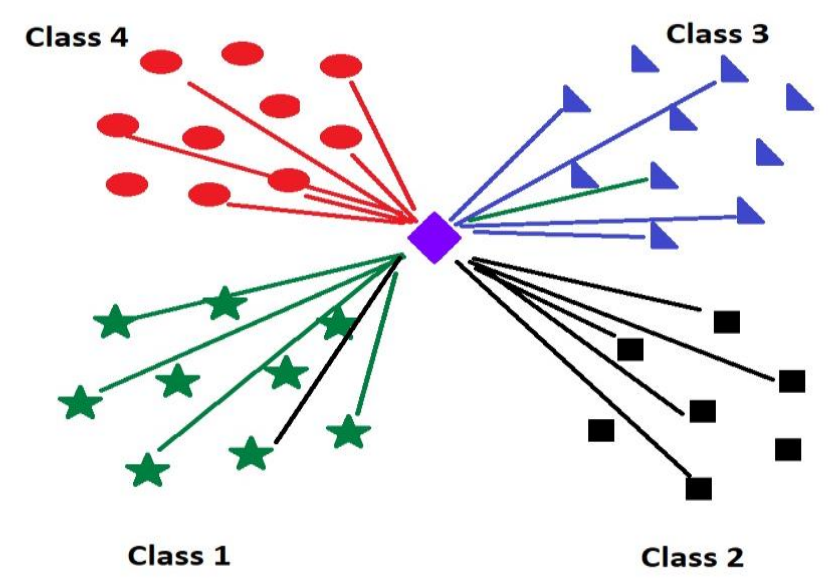

Let $\left\{x_{i}, y_{i}\right\} ; i=1,2, \ldots, n$ be the training sample in which $x_{i}$ represents the feature value and $y_{i} \in\left\{c_{1}, c_{2}, \ldots ., c_{M}\right\}$ represents the $\mathrm{M}$ categories (class value). Furthermore, let $X$ be a novel data point. The kNN algorithm can be summarised as follows.

- Calculating the distance from this novel point $X$ to all other points in the dataset.

- Sort the distances from each point to the novel point and select the $k$ (usually an odd number to prevent tie situations) smallest distances, i.e., nearest $k$ neighbours $y_{i 1}, y_{i 2}, \ldots, y_{i k}$.

- Then for each of the above $k$ nearest neighbours, it records the corresponding class (labels) $c_{j} ; j=1,2, . ., M$ and calculate the following conditional probability. 


$$
P\left(c_{x}=c_{j} \mid X=x\right)=\frac{1}{k} \sum_{i=1}^{k} I_{c_{i}}(x)
$$

where $I_{c_{i}}(x)=\left\{\begin{array}{l}1 ; x \in c_{i} \\ 0 ; x \notin c_{i}\end{array}\right.$

- The class $c_{j}$ that has the highest probability is assigned to the novel data point, as the category of the data point.

Table 3. Popular Distance Metrics

\begin{tabular}{|l|l|}
\hline Name & Distance Matric \\
\hline Euclidean & $\sqrt{\sum_{i=1}^{n}\left(x_{i}-y_{i}\right)^{2}}$ \\
\hline Manhattan & $\sum_{i=1}^{n}\left|x_{i}-y_{i}\right|$ \\
\hline Chebyshev & $\max \left(\left|x_{i}-y_{i}\right|\right)$ \\
\hline Minkowski & $\sqrt[p]{\sum_{i=1}^{n}\left(x_{i}-y_{i}\right)^{p}}$ \\
\hline
\end{tabular}

\section{Random Forest $(R F)$}

RF algorithm extends the idea of decision trees by aggregating higher number of decision trees to reduce the variance of the novel decision tree (Couronné 2018). Each tree is built upon a collection of random variables (features) and a collection of such random trees is called a Random Forest. Dues to the higher classification accuracy, RF is considered as one of the most successful classification algorithms in modern-times (Breiman 2001, Biau and Scornet 2016) (Figure 3). Furthermore, the performance of this classification algorithm is significant for unbalanced and missing data (Shah et al. 2014), compared to its counterparts. RF has been studied by many researchers both in theoretically and experimentally since its introduction in 2001 (Bernard et al. 2007, Breiman 2001, Geurts 2006, Rodriguez 2006). Further studies have been conducted to improve the classify-cation accuracy of RF by clever selection of the associated parameters of RF (Bernard et al. 2007).

A handful of applications of machine learning algorithms in the context of cricket can be seen in the literature. Using $\mathrm{kNN}$ and NB classifiers, Kumar and Roy (2018) forecasted final score of an ODI score after the completion of the fifth over of the game. NB and RF were two of the machine learning techniques Passi and Pandey (2018) used in their study to predict the individual player's performance in the game of cricket. Using English T20 county cricket data from 2009 to 2014, Kampakis and Thomas (2015) developed a machine learning model to predict the outcome of the T20 cricket game. 
Figure 3. Random Forest Classifier
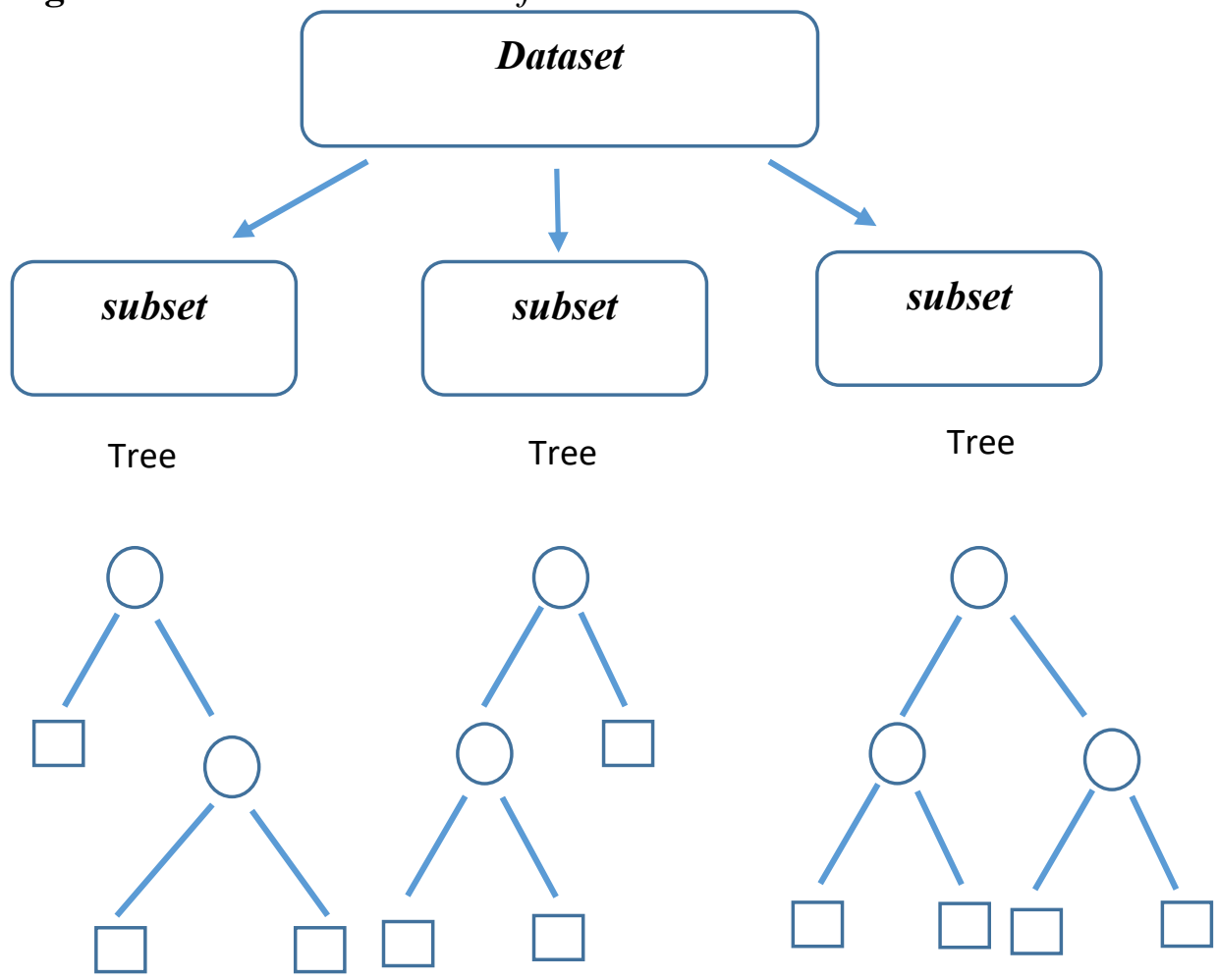

Findings

All the experimental outcomes were tested under the k-fold cross-validation, which is used to generalize the findings of the study to any given independent sample as discussed in the literature (Burman 1989, Kohavi 1995). We executed all of the three machine learning classifiers with the collected data and according to the experimental outcomes, NB classifier reached a maximum of $60.7 \%$ prediction accuracy. Furthermore, the maximum prediction accuracy using Knn was $55.08 \%$. In order to see how the prediction accuracy changes with the selection of distance matric with $\mathrm{kNN}$ algorithm, we measured the prediction of accuracies with respect to the various distance matrices. Table 4 summarises the percentage of prediction accuracy for each of the selected distance metric and the value $k$ used in $\mathrm{kNN}$.

With RF, an initial accuracy rate of $93.34 \%$ was recorded, which is the highest among the three classifiers we used. Further investigation was conducted to optimize the prediction accuracy, by varying the associated parameters of RF. 
Table 4. Distance Metric, K value, and Percentage of Prediction Accuracy

\begin{tabular}{|l|c|c|}
\hline Distance Metric & K & Percentage of Accuracy \\
\hline \multirow{3}{*}{ Euclidian } & 3 & $47.55 \%$ \\
\cline { 2 - 3 } & 5 & $49.36 \%$ \\
\cline { 2 - 3 } & 7 & $46.77 \%$ \\
\hline \multirow{3}{*}{ Manhattan } & 3 & $49.81 \%$ \\
\cline { 2 - 3 } & 5 & $51.98 \%$ \\
\cline { 2 - 3 } & 7 & $54.83 \%$ \\
\cline { 2 - 3 } & 3 & $49.88 \%$ \\
\cline { 2 - 3 } & 5 & $52.38 \%$ \\
\cline { 2 - 3 } & 7 & $55.08 \%$ \\
\cline { 2 - 3 } & 3 & $43.38 \%$ \\
\hline \multirow{3}{*}{ Minkowski, p=3 } & 5 & $50.99 \%$ \\
\hline
\end{tabular}

\section{Important Parameters used in RF}

Among the various parameters used with RF, the following important parameters were changed to see a better prediction rate.

n_estimators: $\quad$ This represents the number of trees in the RF.

max_features: This represents the maximum number of features when the RF selects the split point.

min_samples_leaf: This represents how many minimum number of data points in the end node.

Figure 4. Change of Prediction Accuracy with Different Parameters

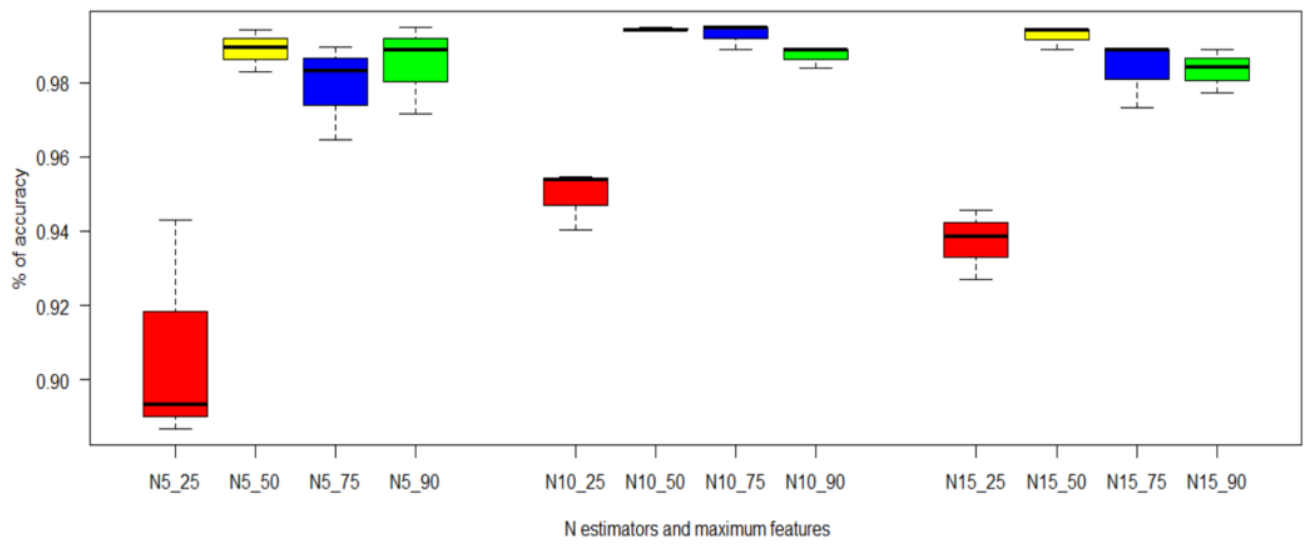

Values of n_estimators, max_features, and min_samples_leaf were varied from $\{5,10,15\},\{0.25,0.50,0.75,0.90\}$, and $\{1,2,3\}$. The obtained corresponding values of the percentages of prediction accuracies are displayed by the Figure 4 .

After searching for better parameterization, we investigated the associated errors with the RF Regression model. Both Mean Absolute Error (MAE) and Mean Squared Error (MSE) were recorded for the model with previously identified parameters. Figures 6 and 7 display the variation of both MAE and MSE 
Vol. 7, No. $1 \quad$ Wickramasinghe: Classification of All-Rounders in the Game of ODI...

for each values of parameters. As the experimental outcomes indicated, RF reached a maximum prediction accuracy of $99.4 \%$ with the selection of $n \_$estimators $=10$, max_features $=0.50$, min_samples_leaf $=2$, and n_estimators $=10$, max_features $=0.75$, min_samples_leaf $=1$.

Figure 5. Prediction Accuracy, max_features and min_samples_leaf for $n_{-}$ estimators $=10$

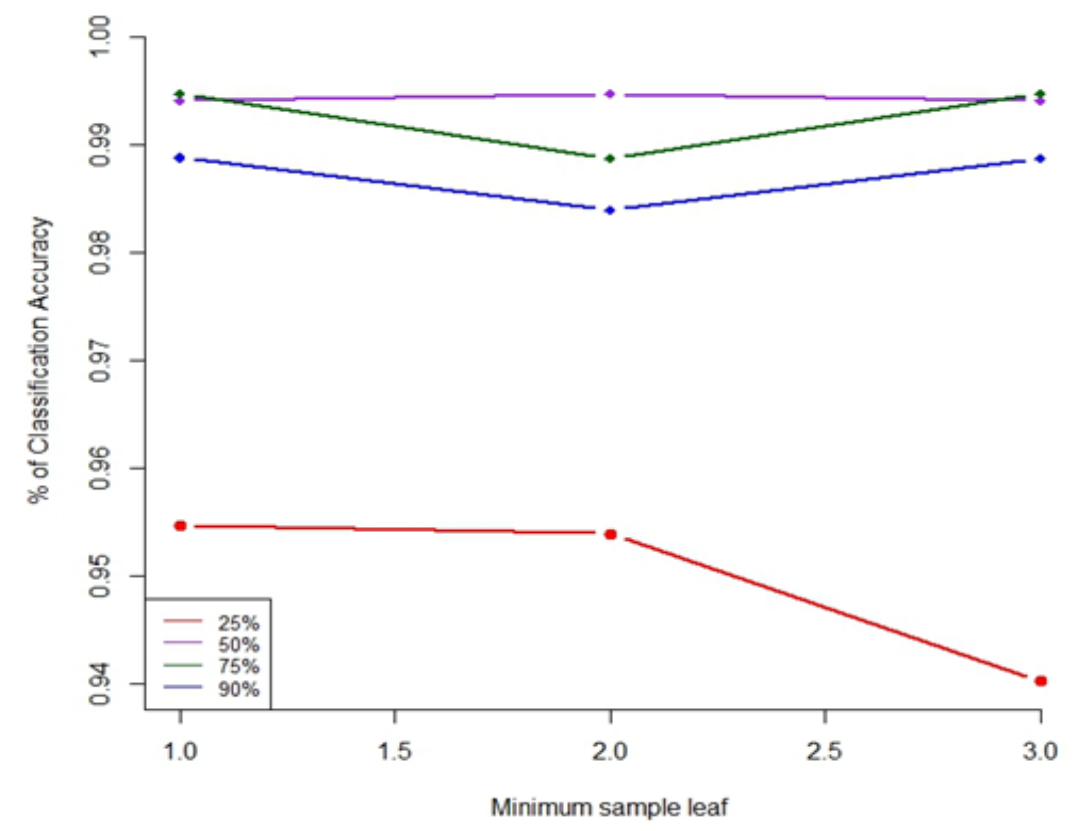

Figure 6. Parameters of Random Forest vs. Mean Absolute Error

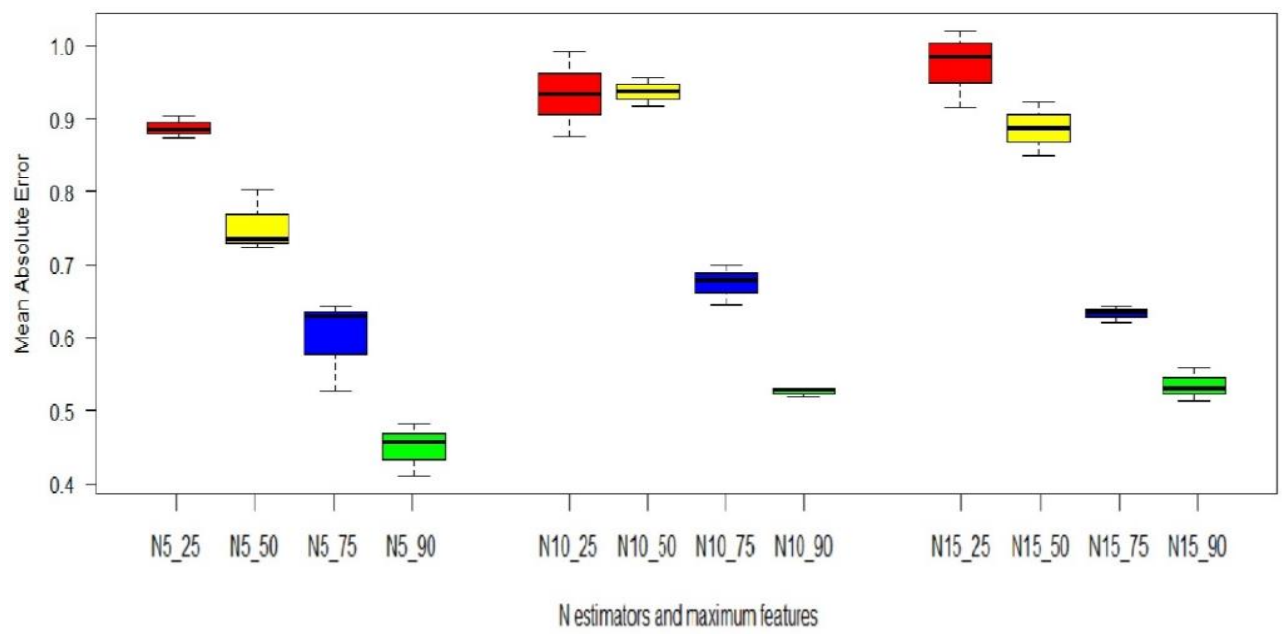


Figure 7. Parameters of Random Forest vs. Mean Square Error

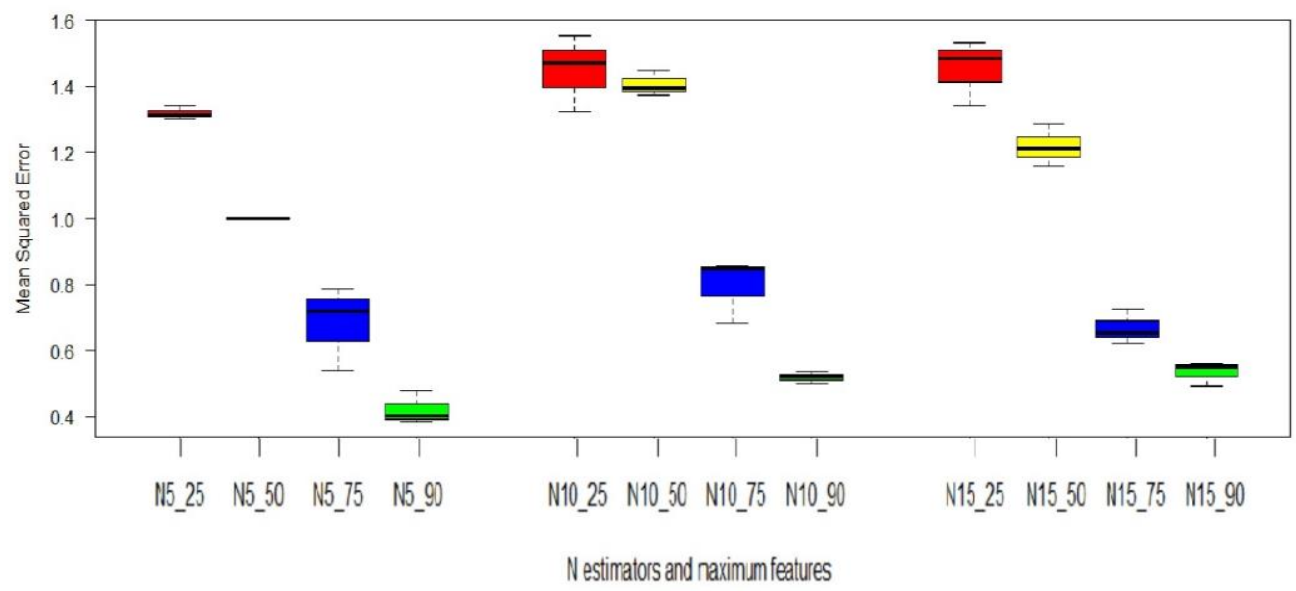

\section{Discussion and Conclusion}

In this manuscript, we discussed how to categorize all-rounders in the game of ODI cricket. Using a collection of 177 players from all the ODI playing countries, ten player-related predictors, together with three machine learning techniques, we investigated how to categorize all-rounders into one of the four categories. In this study, we utilized three machine learning techniques, namely Random Forest $(\mathrm{RF}), \mathrm{k}$-nearest neighbours ( $\mathrm{kNN}$ ), and Naïve Bayes (NB) to predict the appropriate category each of the all-rounder should belong.

After initial execution of the above three algorithms, the prediction accuracies for $\mathrm{kNN}, \mathrm{NB}$, and $\mathrm{RF}$ were $50.08 \%, 59.00 \%$, and $93.34 \%$ respectively. Further improvement of the prediction accuracy was able to achieve with the proper selection of the parameters. By changing the distance metric with $\mathrm{kNN}$ and the $k$ value, we were able to improve the prediction accuracy up to $55.08 \%$. Similarly, NB was improved up to $60.7 \%$. According to Figures 4-7, it is clear that RF has improved to the highest prediction accuracy of $99.4 \%$, with the selection of appropriate values for the parameters. This can be reached with two different parameter settings. i.e., when $n \_$estimators is 10 , max_features is 0.50 , min samples_leaf is 2, and n_estimators is 10 , max_features is 0.75 , min_samples_leaf is 1 . In addition to the prediction accuracy, an investigation was conducted to find out the relative errors involved with these processes. According to the findings, these errors became minimum when n_estimators is 10 , max_features is 0.75 and min_samples_leaf is 1 respectively and the values were 0.68 and 0.86 respectively.

In summary, our experimental results indicated that RF algorithm outperformed both $\mathrm{kNN}$ and NB by huge margins. The findings of this study benefit the officials of the game of cricket and the players in many ways. Player selection committees, coaches of teams, and even the players can utilize these outcomes to identify appropriate all-rounders. It would be important to include 
Vol. 7, No. $1 \quad$ Wickramasinghe: Classification of All-Rounders in the Game of ODI...

additional performance indicators, including statistics about the opposition teams that the players play against for future studies.

\section{References}

Allision PD (1999) Multiple regression: a primer. Thousand oaks. CA: Pine Forge Press.

Arar OF, Ayan K (2017) A feature dependent Naive Bayes approach and its application to the software defectprediction problem. Applied Soft Computing 59(Oct): 197-209.

Bai H, Pan W (2009) An application of a new multivariate resampling method to multiple regression. Multiple Linear Regression Viewpoints 35(1): 1-5.

Bailey T (1989) The greatest since my time. London: Hodder and Stoughton.

Bajramovic F, Mattern F, Butko N, Denzler J (2006) A comparison of nearest neighbor search algorithms for generic object recognition. In J Blanc-Talon, W Philips, D Popescu, P Scheunders (Eds.), Advanced Concepts for Intelligent Vision Systems. ACIVS 2006. Lecture Notes in Computer Science, p. 4179. Berlin, Heidelberg: Springer.

Bandulasiri A, Brown T, Wickramasinghe T (2016) Characterization of the result of one day format of cricket. Operation Research and Decisions 26(4): 21-32.

Bernard S, Heutte L, Adam S (2007) Using random forests for handwritten digit recognition. $9^{\text {th }}$ IAPR/IEEE International Conference on Document Analysis and Recognition (ICDAR), Curitiba, Brazil. 1043-1047, ff10.1109/ICDAR.2007.437707 4ff.ffhal-00436372f.

Biau G, Scornet E (2016) A random forest guided tour. Test 25(2): 197-227. Doi:10.1007/ s11749-016-0481-7.

Breiman L (2001) Random forests. Machine Learning 45(1): 5-32.

Burman P (1989) A comparative study of ordinary cross-validation, v-fold crossvalidation and the repeated learning-testing methods. Biometrika 76(3): 503- 514.

Christie CJA (2012) The physical demands of batting and fast bowling in cricket. In KR Zaslav (Ed.), An International Perspective on Topics in Sports Medicine and Sports Injury: InTech, pp. 321-33.

Couronné R, Probst P, Boulesteix AL (2018) Random forest versus logistic regression: a large-scale benchmark experiment. BMC Bioinformatics 19(1): 270. Doi:10.1186/s 12859-018-2264-5.

Elnahrawy E (2002) Log-based chat room monitoring using text categorization: a comparative study. In St Thomas (Ed.), Proceedings of the IASTED International Conference on Information and Knowledge Sharing (IKS 2002). US Virgin Islands, USA.

Gakis K, Parsalos P, Park J (2016) A probabilistic model for multi-contestant races. Athens Journal of Sports 3(2): 111-118.

Geurts G, Ernst D, Wehenkel L (2006) Extremely randomized trees. Machine Learning 36(1): 3-42.

Hand DJ (1992) Statistical methods in diagnosis. Statistical Methods in Medical Research 1(1): 49-67.

Kampakis S, Thomas B (2015) Using machine learning to predict the outcome of English county twenty over cricket matches. Arxiv: Machine Learning, 1-17.

Kohavi R (1995) A study of cross-validation and bootstrap for accuracy estimation and model selection. Proceedings of the $14^{\text {th }}$ Joint Conference on Artificial Intelligence (IJCAI), vol. 2, 1137-1143. 
Kumar S, Roy S (2018) Score prediction and player classification model in the game of cricket using machine learning. International Journal of Scientific \& Engineering Research 9(8): 237-242.

Kuncheva LI (2006). On the optimality of Naive Bayes with dependent binary features. Pattern Recognition Letters 27(7): 830-837.

Passi K, Pandey N (2018) Increased prediction accuracy in the game of cricket using machine learning. International Journal of Data Mining \& Knowledge Management Process 8(2): 19-36.

Rodriguez J, Kuncheva L, Alonso C (2006) Rotation forest: A new classifier ensemble method. IEEE Transactions on Pattern Analysis and Machine Intelligence 28(10): 1619-1630.

Saikia H, Bhattacharjee D (2011) On classification of all-rounders of the Indian premier league (IPL): a Bayesian approach. Vikalpa 36(4): 51-66. 10.1177/02560909201104 04.

Saikia H, Bhattacharjee D, Radhakrishnan UK (2016) A new model for player selection in cricket. International Journal of Performance Analysis in Sport 16(Apr): 373-388.

Shah AD, Bartlett JW, Carpenter J, Nicholas O, Hemingway H (2014) Comparison of random forest and parametric imputation models for imputing missing data using mice: a caliber study. American Journal of Epidemiology 179(6): 764-774. https:// doi.org/10.1093/aje/kwt312.

Stevenson OG, Brewer BJ (2019) Modelling career trajectories of cricket players using Gaussian processes. In R Argiento, D Durante, S Wade (Eds.), Bayesian Statistics and New Generations. BAYSM 2018. Springer Proceedings in Mathematics \& Statistics, vol. 296. Springer, Cham.

Tan A, Ramachandran R (2010) Ranking the greatest all-rounders in test cricket. Available at: www.cricketsociety.com/ranking_the_greatest_all-ro.pdf.

Van Staden PJ (2008) Comparison of bowlers, batsmen and all-rounders in cricket using graphical display. Technical Report 08/01. Department of Statistics, University of Pretoria, South Africa.

Wickramasinghe IP (2014) Predicting the performance of batsmen in test cricket. Journal of Human Sport \& Exercise 9(4): 744-751.

Wu X, Kumar V, Quinlan R, Ghosh J, Yang Q, Motoda H, McLachlan J, Ng A, Liu B, Yu PS, Zhou Z, Steinbach M, Hand DJ, Steinberg D (2008) Top 10 algorithms in data mining. Knowledge and Information Systems 14(1): 1-37.

Xiubo G, Liu T, Qin T, Arnold A, Li H, Shum H (2008) Query dependent ranking using $\mathrm{K}$-nearest neighbor, Proceedings of the $31^{\text {st }}$ Annual International ACM SIGIR Conference on Research and Development in Information Retrieval. Singapore. Doi:10.1145/1390334.1390356. 
